\title{
Introduction to this Special Issue
}

\section{"Nonlinear waves and chaos in space plasmas"}

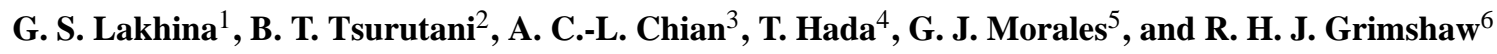 \\ ${ }^{1}$ Indian Institute of Geomagnetism, New Panvel (W), Navi Mumbai, India \\ ${ }^{2}$ Jet Propulsion Laboratory, California Institute of Technology, Pasadena, CA, USA \\ ${ }^{3}$ Paris Observatory-LESIA, Meudon, France and National Institute for Space Research (INPE), Sao Jose dos Campos, Brazil \\ ${ }^{4}$ Interdisciplinary Graduate School of Engineering Sciences, Kyushu University, Fukuoka, 816-8580, Japan \\ ${ }^{5}$ Physics and Astronomy Department, University of California, Los Angeles, CA 90095, USA \\ ${ }^{6}$ Loughborough University, Department of Mathematical Sciences, Ashby Road, Loughborough, LE11 3TU, UK
}

Correspondence to: G. S. Lakhina (gslakhina@gmail.com)

Nonlinear waves and chaos (NWC) are believed to play dominant roles in the heating and acceleration of charged particles and generating turbulence in space, astrophysical and laboratory plasmas. A series of workshops on "Nonlinear Waves and Chaos in Space Plasmas" have been held to discuss this subject: in Kyoto, Japan (1994), Köln, Germany (1997), Carlsbad, USA (1999), Tromsø, Norway (2001), Mumbai, India (2003), Fukuoka, Japan (2006), Beaulieu-sur-Mer, France (2008), and La Jolla, USA (2010). The ninth NWC workshop 2013 of this series was held in La Jolla, USA, 3-8 March 2013.

This workshop brought together 31 space plasma, laboratory plasma, and fusion physicists, astrophysicists and atmospheric physicists from various countries. The workshop provided an opportunity to report on the latest results from theory, simulation and data analysis dealing with the nonlinear processes and complex system dynamics in space, laboratory and astrophysical plasmas as well as nonlinear interaction between large-scale waves in the Earth's atmosphere.

This special issue of Nonlinear Processes in Geophysics (NPG) consists of papers drawn from the talks given at NWCW 2013 as well as papers that were submitted in response to the call for the papers for this special issue. All papers had to undergo the usual NPG review process before they were finally accepted for this special issue.

The papers cover a broad range of topics related to nonlinear waves and chaos. These include the multifractal spectrum in the heliosphere and the heliosheath, chaoticity and dynamical complexity in the ionosphere, Alfvén waves in space and astrophysical dusty plasmas, nonlinear electromagnetic waves in magnetized relativistic plasmas, ion acceleration by nonlinear Alfvén wave packets, the relativistic surfatron process for Landau resonant electrons in radiation belts, stochastic electron motion driven by nonlinear waves, turbulence in the interstellar medium, four-dimensional energy spectrum for space-time structure of plasma turbulence, and validity of Taylor's hypothesis under large-scale flow variations. A brief summary of the special issue papers is given below.

Macek and Wawrzaszek (2014) report on the multifractal scaling of the fluctuations in the interplanetary magnetic field strength as measured onboard Voyager 2 in the heliosphere and the heliosheath. They find that the spectrum is prevalently right-skewed and the degree of multifractality is modulated by the solar activity inside the heliosphere. The results seem to support the idea that the solar wind termination shock is asymmetric.

Ogunsua et al. (2014) analyze total electron content (TEC) time series data during the year 2011 obtained by three global positioning system (GPS) receivers within Nigeria, which lies within the equatorial ionization anomaly region. They find positive Lyapunov exponents (LE) for both quiet and disturbed days, which indicates chaoticity in the system. For different days, the chaoticity of the ionosphere exhibits no definite pattern for either quiet or disturbed days. However, values of the LE were lower for the storm period compared with its nearest relatively quiet periods for all the stations. The monthly averages of LE and entropy also show no definite pattern. The values of the computed correlation dimension range from 2.8 to 3.5 , with the lowest values recorded during the storm period of October 2011. 
Jatenco-Pereira et al. (2014) present results on the effects of superthermal particles on the dispersive characteristics of Alfvén waves in dusty plasmas in different astrophysical and space regions. It is shown that the presence of dust and superthermal particles significantly modifies the dispersion of Alfvén waves. The authors discuss the competition between different damping processes of kinetic Alfvén and Alfvén cyclotron waves. They briefly review the nonlinear evolution of Alfvén waves to chaos. Some applications of Alfvén waves in the auroral region of space plasmas, as well as stellar winds and star-forming regions of astrophysical plasmas are discussed.

Muñoz et al. (2014) have shown that for large-amplitude, circularly polarized waves propagating along a constant magnetic field, an exact solution of the fluid equations can be derived for relativistic temperatures. It is found that relativistic thermal effects produce a decrease in both the effective plasma frequency and the upper frequency cutoff for the Alfvén branch. It is also found that the Alfvén speed decreases with temperature, being zero for infinite temperature. The authors also studied the same system based on the relativistic Vlasov equation, including thermal effects along the direction of propagation. It is concluded that kinetic and fluid results are qualitatively consistent, but have several quantitative differences.

The paper by Nariyuki et al. (2014) reports the nonlinear evolution of parallel propagating Alfvén waves in a radially expanding plasma from numerical simulations of a kineticfluid model. It is found that the steepened Alfvén wave packets outwardly accelerate ions, which can be observed as the beam components in the interplanetary space. The energy of imposed Alfvén waves is converted into longitudinal fluctuations by nonlinear steepening and nonlinear Landau damping. Wave shoaling due to the inhomogeneity of the phase velocity is also observed.

Osmane and Hamza (2014) report new results on the relativistic surfatron process for Landau resonant electrons in radiation belts. Their model includes longitudinal wave effects and inhomogeneous magnetic fields. It is found that even though both effects can limit the surfatron acceleration of electrons in the radiation belts, gains in energy of the order of $100 \mathrm{keV}$ can occur in $10^{-4} \mathrm{~s}$. The authors conclude that this is sufficiently strong to be relevant to radiation belt dynamics.

The paper by Khazanov et al. (2014) deals with stochastic electron motion driven by various nonlinear waves. It is found that the interaction between whistler-mode waves and radiation-belt electrons is likely to involve the same mechanism that is responsible for the dynamical balance between the accelerating process and relativistic electron precipitation events. The paper discusses the efficiency of the stochastic surfing acceleration mechanism for cosmic electrons at supernova remnant shock fronts involving Langmuir wave packets. Analytical and numerical methods for studying Hamiltonian chaos and dissipative strange attractors, and for determining the heating extent and energy spectra, are discussed.

Falceta-Gonçalves et al. (2014) give a review of interstellar turbulence focusing on the statistics of its structure and energy cascade. The physics of compressible and incompressible turbulent flows in magnetized plasmas is discussed. The most relevant observational techniques providing quantitative insights of interstellar turbulence are also presented. The main difficulties in developing a three-dimensional view of interstellar turbulence from these observations as well as the main sources of interstellar turbulence are briefly reviewed.

The paper by Narita (2014) discusses a four-dimensional energy spectrum for the space-time structure of plasma turbulence. The model is based on the Eulerian wave numberfrequency spectrum developed for describing fluid turbulence. This includes wave vector anisotropies in the threedimensional wave vector domain by approximating the spectrum to a set of ellipses. The model is applied to the magnetic energy spectrum in the near-Earth solar wind measured by four Cluster spacecraft, and the set of the spectral parameters are determined observationally. In this way, the spacetime structure of plasma turbulence can be condensed into a small number of parameters, suitable for evaluating the energy spectra in observational and numerical studies on a quantitative basis.

The paper by Wilczek et al. (2014) discusses the validity of Taylor's hypothesis under large-scale flow variation based on a simple model for the wavenumber-frequency spectrum that has recently been introduced as a generalization of Kraichnan's random sweeping hypothesis. For turbulence with a power-law energy spectrum, it is found that the wavenumber spectrum estimated by Taylor's hypothesis exhibits the same power-law as the true spectrum, but the spectral energy is overestimated due to the large-scale flow variation.

We deeply thank all the referees who donated their time to carefully review the papers and give valuable suggestions/comments which led to improvement of the papers. We hope that the readers of NPG will enjoy this special issue.

\section{References}

Falceta-Gonçalves, D., Kowal, G., Falgarone, E. and Chian, A. C.L.: Turbulence in the Interstellar Medium, Nonlin. Processes Geophys., accepted, 2014.

Jatenco-Pereira, V., Chian, A. C.-L., and Rubab, N.: Alfvén waves in space and astrophysical dusty plasmas, Nonlin. Processes Geophys., 21, 405-416, doi:10.5194/npg-21-405-2014, 2014.

Khazanov, G. V., Tel'nikhin, A. A., and Kronberg, T. K.: Stochastic electron motion driven by space plasma waves, Nonlin. Processes Geophys., 21, 61-85, doi:10.5194/npg-21-61-2014, 2014.

Macek, W. M. and Wawrzaszek, A.: Voyager 2 observation of the multifractal spectrum in the heliosphere and the heliosheath, Nonlin. Processes Geophys., 20, 1061-1070, doi:10.5194/npg20-1061-2013, 2013. 
Muñoz, V., Asenjo, F. A., Domínguez, M., López, R. A., Valdivia, J. A., Viñas, A., and Hada, T.: Large-amplitude electromagnetic waves in magnetized relativistic plasmas with temperature, Nonlin. Processes Geophys., 21, 217-236, doi:10.5194/npg-21-2172014, 2014.

Narita, Y.: Four-dimensional energy spectrum for space-time structure of plasma turbulence, Nonlin. Processes Geophys., 21, 4147, doi:10.5194/npg-21-41-2014, 2014.

Nariyuki, Y., Umeda, T., Suzuki, T. K., and Hada, T.: Ion acceleration by parallel propagating nonlinear Alfvén wave packets in a radially expanding plasma, Nonlin. Processes Geophys., 21, 339-346, doi:10.5194/npg-21-339-2014, 2014.
Ogunsua, B. O., Laoye, J. A., Fuwape, I. A., and Rabiu, A. B.: The comparative study of chaoticity and dynamical complexity of the low-latitude ionosphere, over Nigeria, during quiet and disturbed days, Nonlin. Processes Geophys., 21, 127-142, doi:10.5194/npg-21-127-2014, 2014.

Osmane, A. and Hamza, A. M.: Relativistic surfatron process for Landau resonant electrons in radiation belts, Nonlin. Processes Geophys., 21, 115-125, doi:10.5194/npg-21-115-2014, 2014.

Wilczek, M., Xu, H. and Narita, Y.: A note on Taylor's hypothesis under large-scale flow variation, Nonlin. Processes Geophys. accepted, 2014. 